\title{
BOUNDS ON LEAST DILATATIONS
}

\author{
R. C. PENNER
}

(Communicated by Irwin Kra)

\begin{abstract}
We consider the collection of all pseudo-Anosov homeomorphisms on a surface of fixed topological type. To each such homeomorphism is associated a real-valued invariant, called its dilatation (which is greater than one), and we define the spectrum of the surface to be the collection of logarithms of dilatations of pseudo-Anosov maps supported on the surface. The spectrum is a natural object of study from the topological, geometric, and dynamical points of view. We are concerned in this paper with the least element of the spectrum, and explicit upper and lower bounds on this least element are derived in terms of the topological type of the surface; train tracks are the main tool used in establishing our estimates.
\end{abstract}

\section{INTRODUCTION}

Fix a surface $F_{g}^{s}$ of genus $g$ with $s$ distinguished points and empty boundary, where we assume that $2 g-2+s>0$ and $s \geq 0$. A map $\phi: F_{g}^{s} \rightarrow F_{g}^{s}$ preserving the distinguished points setwise is said to be pseudo-Anosov (to be abbreviated pA) if there are a pair $\mathscr{F}_{ \pm}$of transverse measured foliations of $F_{g}^{s}$ with $\phi\left(\mathscr{F}_{ \pm}\right)=\lambda^{ \pm 1} \mathscr{F}_{ \pm}$for some $\lambda>1$. (See [FLP], for instance, for definitions and basic properties of measured foliations and pA maps.) The number $\lambda$ is called the dilatation of $\phi$, and we define the spectrum of $F_{g}^{s}$ to be

$$
\sum_{g}^{s}=\left\{\log \lambda: \lambda \text { is the dilatation of a pA map of } F_{g}^{s}\right\} .
$$

$\sum_{g}^{s}$ can also be described as the set of Teichmüller lengths of Teichmüller geodesics on the moduli space of $F_{g}^{s}$ (see [Ab] for example), or alternatively, as the set of topological entropies of pA automorphisms of $F_{g}^{s}$ (see [FLP]). An elementary argument (see [AY]) shows that $\sum_{g}^{s} \subset \mathbb{R}$ is discrete.

In this paper, we give both upper and lower bounds to the least element $l_{g}^{s}$

Received by the editors October 21, 1986 and, in revised form, August 21, 1990. 1980 Mathematics Subject Classification (1985 Revision). Primary 57N06, 57N50.

Partially supported by grants from the National Science Foundation and the Maxwell Research Foundation. 
of $\sum_{g}^{s}$. In fact, we shall find that

and

$$
l_{g}^{s} \geq \frac{\log 2}{12 g-12+4 s},
$$

$$
\frac{\log 1}{g}-\geq l_{g}^{0} \geq \frac{\log 2}{12 g-12} .
$$

The lower bounds are established by employing the train track theory and a simple (but apparently not well known) spectral theory trick. The upper bound comes about by applying the main result of [P1] to give examples of $\mathrm{pA}$ maps on closed surfaces. The results of [Ba] can be used to sharpen the upper bound when $s=0$, and we expect they can also be used to give an analogous upper bound in case $s \neq 0$.

\section{NOTATION AND BACKGROUND}

To establish notation, we quickly recall the relevant train track theory. This material is discussed more fully in $[\mathrm{Pa}, \mathrm{P} 1, \mathrm{PH}, \mathrm{PP}, \mathrm{Th}]$; the necessary background information is fully covered in [PP, P1]. In particular, we recall that the train track $\tau$ is said to be recurrent if it supports a strictly positive transverse measure, and it is said to be trivalent provided that each switch has valence exactly three. $\tau$ fills $F_{g}^{s}$ if each component of $F_{g}^{s}-\tau$ is a (perhaps oncepunctured) topological disk.

Suppose that $\sigma$ and $\tau$ are train tracks in the surface $F_{g}^{s}$. If $\sigma$ is smoothly homotopic into $\tau$, then we say that $\tau$ carries $\sigma$, and we write $\sigma<\tau$; notice that the relation $<$ is transitive. In particular, if $\phi$ is a pA map of $F_{g}^{s}$, then (as shown in [PP, Theorem 4.1]) there is a trivalent and recurrent train track $\tau \subset F_{g}^{s}$ so that $\phi(\tau)<\tau$. Given a branch of the train track $\tau$, as above, we may choose a fiber, called the central tie over the branch, in the tie-neighborhood over the branch. Suppose that the train track $\tau$ has branches $\left\{b_{i}\right\}_{i}^{n}$ and define an $n$-by$n$ matrix $M=\left(M_{i j}\right)$, called the incidence matrix (for the carrying), as follows: since $\tau$ carries $\phi(\tau)$, there is a smooth map $h: F \rightarrow F$ which homotopes $\phi(\tau)$ into the tie-neighborhood of $\tau$ keeping the train tracks transverse to the ties, and we choose such an $h$, called a "supporting map for the carrying" $\tau>\phi(\tau)$; $M_{i j}$ is the number of intersections (counted with unsigned multiplicity) of the image of $h \circ \phi\left(b_{i}\right)$ with the central tie over $b_{j}$. (Thus, the incidence matrix evidently depends on the choice of a supporting map.)

The cone $V_{\tau}$ of transverse measures on $\tau$ is identified with a corresponding cone in the space $\mathscr{M} \mathscr{F}_{0}\left(F_{g}^{s}\right)$ of compactly supported measured foliations on $F_{g}^{S}$ (see [P1, p. 181]), and any incidence matrix (for any choice of supporting map) $M$ describes the action of $\phi$ on $V_{\tau}$. If $\tau$ and the carrying of $\phi(\tau)$ by $\tau$ are chosen carefully, then the incidence matrix $M$ is Perron-Frobenius (to be abbreviated PF) in the sense that $M$ has nonnegative entries and some iterate of $M$ has strictly positive entries; see [PP, Theorem 4.1]. (See [Ga] for a general discussion of PF matrices, which are called "primitive irreducible" there.) The unique positive eigenvector of $M$ corresponds to the unstable foliation $\mathscr{F}_{+}$of 
$\phi$ (see [P1, p. 189]), and it follows that the dilatation of $\phi$ is simply the spectral radius of $M$.

\section{THE LOWER BOUND}

We begin with an easy estimate on the spectral radii of certain PF matrices.

Lemma. Suppose that $A$ is an $n$-by-n integral PF matrix, where $n>1$. If $\lambda$ denotes the spectral radius of $A$, then

$$
\log \lambda \geq \log 2 / n .
$$

Proof. Suppose that the vector $\vec{x} \in \mathbb{R}^{n}$ has coordinates $\left(x_{i}\right)_{1}^{n}$, and consider the function

$$
|\vec{x}|=\sum_{1}^{n} x_{i}
$$

defined on the "cone"

$$
C=\left\{\vec{x} \in \mathbb{R}^{n}: x_{i} \geq 0 \text { for each } i \text { and } \vec{x} \neq \overrightarrow{0}\right\} .
$$

We claim that for any $\vec{x} \in C$, we have

$$
\left|A^{n} \vec{x}\right| /|\vec{x}| \geq 2 .
$$

The lemma follows easily from this claim since the eigenvector of $A$ corresponding to $\lambda$ itself lies in (the interior of) $C$. By linearity of the norm, the claim will follow once we show that $\left|A^{n} \vec{e}\right| \geq 2$ for any unit coordinate vector $\vec{e} \in C$.

Given a matrix $A^{n \times n}$ with nonnegative entries, we define the oriented graph $G=G(A)$ of $A$ to be the graph on $n$ vertices with an oriented edge running from the $i$ th vertex to the $j$ th vertex if $A_{i j} \neq 0$. If $A^{m}=\left(A_{i j}^{m}\right)$, then $A_{i j}^{m}$ is exactly the number oriented edge-paths in $G$ of length $m$ from the $i$ th to the $j$ th vertex.

Since we assume that $A$ is $\mathrm{PF}$, some power of $A$ has strictly positive entries, so in particular, the oriented graph $G$ of $A$ is strongly connected (i.e., for any pair of vertices $v_{1}$ and $v_{2}$, there is an oriented path in $G$ from $v_{1}$ to $v_{2}$ ).

It follows that for each vertex $v$ of $G$ there are at least two distinct oriented paths in $G$ of length $n$ starting from $v$, for otherwise $G$ has the topological type of a circle, so that $A$ is a permutation matrix, contradicting our assumption that $A$ is PF. Thus, $\left|A^{n} \vec{e}\right| \geq 2$ as was claimed, and the proof is complete.

Suppose that $\phi$ is a pA map of $F_{g}^{s}$. It is well known (see for instance [PP, Theorem 4.1]) that there is some trivalent and recurrent train track $\tau$ filling $F_{g}^{s}$ so that $\phi(\tau)<\tau$ with PF incidence matrix. Insofar as the spectral radius of the incidence matrix is the dilatation of the pA map, we might bound above the number of branches of $\tau$ and apply the lemma to obtain a lower bound on the dilatation of $\phi$. This estimate can be improved, however (by roughly $\frac{3}{2}$ ), using some techniques which we introduce presently. 


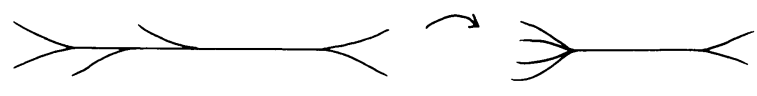

Figure 1

A branch $b$ of a recurrent trivalent train track $\tau$ is said to be large at an endpoint $w$ if every smooth path in $\tau$ through $w$ meets the interior of $b$; otherwise $b$ is called small at $w$. A branch is called simply large (small, respectively) if it is large (small) at both its endpoints; branches that are neither small nor large are called mixed. A mixed branch has a natural orientation starting at its large endpoint.

We claim that there can be no imbedded closed edge-path on a recurrent train track consisting entirely of mixed branches. Such an edge-path $c$ inherits an orientation from the orientation on each of its mixed branches. If $\mu$ is a positive measure on $\tau$, then the $\mu$-weight of branches must strictly increase as we traverse $c$ in the prescribed orientation, and this is evidently impossible.

It follows easily that if $\tau \subset F_{g}^{s}$ is a recurrent and trivalent train track, then each component of

\section{$\bigcup\{$ mixed branches of $\tau\}$}

is contractible.

Now, suppose that $\phi: F_{g}^{s} \rightarrow F_{g}^{s}$ is $\mathrm{pA}$, and let $\tau$ be a trivalent and recurrent train track so that $\phi(\tau)<\tau$. Consider the train track $\sigma$, arrived at by collapsing each component of $\bigcup\{$ mixed branches of $\tau\}$ to a point; see Figure 1. By construction, $\sigma$ has no mixed branches, but the switches of $\sigma$ may have very large valence. A measure on $\sigma$ is determined by its values on the small branches, and there is one linear constraint arising from each large branch; these constraints are not necessarily independent.

Since $\sigma<\tau, \tau<\sigma$, and $\phi(\tau)<\tau$ by construction, we find

$$
\phi(\sigma)<\phi(\tau)<\tau<\sigma
$$

and one easily isotopes $\phi$ transversely to the fibers of the tie-neighborhood of $\tau$ to get a map (of the same name) so that an incidence matrix of the supporting map of the carrying $\phi(\sigma)<\sigma$ is PF.

To proceed, we furthermore isotope $\phi$ so that the image of a large branch of $\sigma$ is contained in the tie neighborhood about a large branch of $\sigma$. To accomplish this, if $b$ is a large branch of $\sigma$, then there are two possibilities: either $\phi(b)$ meets the central tie of a large branch of $\sigma$, or $\phi(b)$ is contained entirely in the tie neighborhood of a single small branch of $\sigma$. In the former case, we can isotope $\phi(\tau)$ transversely to the ties and shrink $\phi(\tau)$ as in Figure $2 a$, and in the latter case, we isotope $\phi(b)$ as in Figure $2 b$.

Consider the small incidence matrix that records the intersections of small branches of $\phi(\sigma)$ with central ties of small branches of $\sigma$. By construction, 


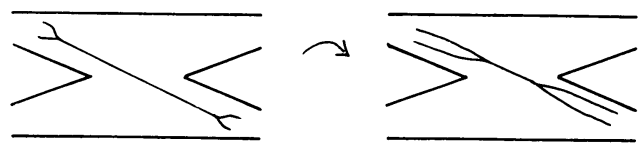

Figure 2a

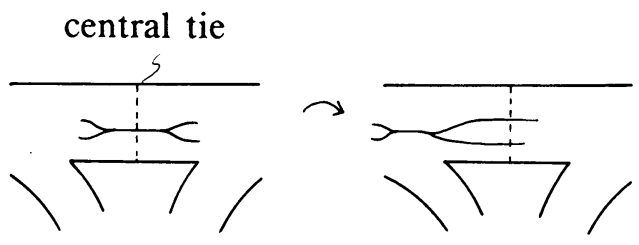

FIGURE 2b

the small incidence matrix is PF and describes the action of $\phi$ on measured foliations carried by $\sigma$.

Theorem. We have the estimate

$$
l_{g}^{s} \geq \frac{\log 2}{12 g-12+4 s} .
$$

Proof. Adopt the notation above, so that $\phi: F_{g}^{s} \rightarrow F_{g}^{s}$ is a pA map, $\tau \subset F_{g}^{s}$ is a trivalent recurrent train track so that $\phi(\tau)<\tau$, and $\sigma \subset F_{g}^{s}$ arises from $\tau$ by collapsing the mixed branches. We bound above the number of small branches of $\sigma$ and apply the lemma to the small incidence matrix. To this end, suppose that $\tau$ has $\beta_{s}$ small and $\beta_{l}$ large branches, let $\beta \geq \beta_{s}+\beta_{l}$ denote the total number of branches of $\tau$, and let $r$ denote the number of switches. Of course, since $\tau$ is trivalent, we have $3 r=2 \beta$, and since there is one linear condition on measures for each switch of $\tau$, we find that $\operatorname{dim}_{\mathbb{R}} V_{\tau} \geq \beta-r$. Thus,

$$
6 g-6+2 s=\operatorname{dim}_{\mathbb{R}} \mathscr{M} \mathscr{F}_{0}\left(F_{g}^{s}\right) \geq \operatorname{dim}_{\mathbb{R}} V_{\tau} \geq \beta-r=\beta / 3,
$$

whence

$$
\beta_{s}+\beta_{l} \leq \beta \leq 18 g-18+6 s .
$$

Furthermore, since there is one linear condition on measures for each large branch of $\sigma$, we conclude

$$
\beta_{s}-\beta_{l} \leq \operatorname{dim}_{\mathbb{R}} V_{\sigma} \leq 6 g-6+2 s,
$$

and arithmetic gives

$$
\beta_{s} \leq 12 g-12+4 s \text {. }
$$

Since the small incidence matrix for the carrying $\phi(\sigma)<\sigma$ is PF of dimension at most $\beta_{s}$ and its spectral radius is the dilatation of $\phi$, the lemma gives the asserted estimate. 
We remark that one would hope to sharpen the bounds of this section using the fact that a homeomorphism of $F_{g}^{s}$ induces a map on $\mathscr{M}_{0}\left(F_{g}^{s}\right)$ which preserves both the geometric intersection pairing and especially the symplectic pairing. (See $[\mathrm{PH}]$ or $[\mathrm{Pa}]$ for instance.)

\section{AN UPPER BOUND BY EXAMPLE}

Consider the surface $F_{g}^{0}, g \geq 2$, as a sphere with $g$ symmetrically placed handles, let $L$ denote the axis of the natural $g$-fold symmetry, and let $a, b, c$ be the simple closed curves indicated in Figure 3a. Let $\rho$ denote rotation by $2 \pi / g$ about $L, \tau_{x}$ denote the righthand twist along the curve $x$, and consider the map

$$
\phi=\rho \circ \tau_{c} \circ \tau_{\alpha}^{-1} \circ \tau_{b}
$$

It follows immediately from [P1, Theorem 2.1$]$ that the $g$ th iterate $\phi^{g}$ is pA, and hence $\phi$ itself is also pA. A train track $\tau \subset F_{g}^{0}$ so that $\phi(\tau)<\tau$ is illustrated in Figure $3 \mathrm{~b}$.

Let $x_{i}, i=1, \ldots, 3 g$, denote the curves $a, b, c, \rho(a), \rho(b), \rho(c), \rho^{2}(a)$, $\ldots, \rho^{g-1}(b), \rho^{g-1}(c)$ respectively. Since $x_{i}$ is carried by $\tau$, there is an associated integral measure $\mu_{i}$ on $\tau$, for $i=1, \ldots, 3 g$. Furthermore, since $\phi\left(x_{i}\right)$ is also carried by $\tau$, for $i=1, \ldots, 3 g$ (as in [P1, p. 194]), the cone $H \subset V_{\tau}$ spanned by $\left\{\mu_{i}\right\}_{1}^{3 g}$ is invariant under $\phi$, and the unstable foliation of $\phi$ is contained in $H$. By expressing the measure on $\tau$ associated to $\phi\left(x_{j}\right)$ as a linear combination of $\left\{\mu_{i}\right\}_{i=1}^{3 g}$, for each $j=1, \ldots, 3 g$, one easily computes the action of $\phi$ on $H$ with respect to the spanning vectors $\mu_{1}, \mu_{2}, \ldots, \mu_{3 g}$

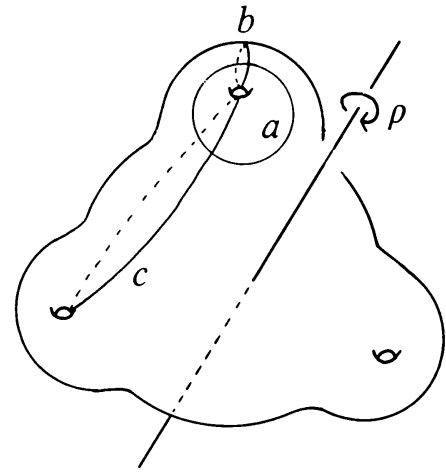

FIGURE 3a

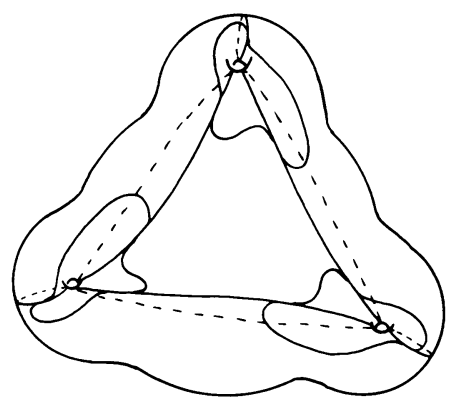

FIGURE 3b 
(in this order) to be

$$
M=\left(\begin{array}{cccccc}
0 & 0 & 0 & \cdots & 0 & I \\
A & B & 0 & \cdots & 0 & C \\
0 & I & 0 & \cdots & 0 & 0 \\
0 & 0 & I & \cdots & 0 & 0 \\
& & \vdots & & & \\
0 & 0 & 0 & \cdots & I & 0
\end{array}\right)
$$

where $I$ denotes the 3-by-3 identity matrix, 0 denotes the 3-by-3 zero matrix, and

$$
A=\left(\begin{array}{lll}
2 & 1 & 1 \\
1 & 1 & 0 \\
2 & 1 & 2
\end{array}\right), \quad B=\left(\begin{array}{lll}
0 & 0 & 0 \\
0 & 0 & 0 \\
1 & 0 & 0
\end{array}\right), \quad \text { and } \quad C=\left(\begin{array}{lll}
0 & 0 & 1 \\
0 & 0 & 0 \\
0 & 0 & 1
\end{array}\right) \text {. }
$$

We claim that (provided $g \geq 4$ ) the $g$ th power of $M$ is given by

$$
\begin{gathered}
M^{g}=\left(\begin{array}{ccccccccc}
A & B & 0 & 0 & \cdots & 0 & 0 & 0 & C \\
C A & D+C B & B A & 0 & \cdots & 0 & 0 & 0 & C^{2} \\
0 & C & D & B A & \cdots & 0 & 0 & 0 & 0 \\
& & & & \vdots & & & \\
0 & 0 & 0 & 0 & \cdots & 0 & C & D & B A \\
B A & 0 & 0 & 0 & \cdots & 0 & 0 & C & D
\end{array}\right), \\
\\
0
\end{gathered}
$$

To prove the claim, first prove by induction that, for $1<k<g$,

$$
M^{k}\left(\begin{array}{cccccccccccc}
0 & \cdots & 0 & 0 & 0 & I & 0 & 0 & 0 & \cdots & 0 & 0 \\
0 & \cdots & 0 & C & D & B A & 0 & \cdots & 0 & 0 & \cdots & 0 \\
0 & \cdots & 0 & 0 & C & D & B A & 0 & \cdots & 0 & 0 & 0 \\
\vdots & & & & & & & & & & & \vdots \\
B A & & & & \cdots & & & & & & C & D \\
A & B & 0 & \cdots & & & & & & \cdots & 0 & C \\
0 & I & 0 & \cdots & & & & & & & \cdots & 0 \\
0 & 0 & I & 0 & \cdots & & & & & & \cdots & 0 \\
\vdots & & & & & & & & & & & \vdots
\end{array}\right) .
$$

Here the first $g-k$ blocks in the first row (of blocks) are 0 ; the next $k-1$ rows (of blocks) have the blocks $C, D$, and $B A$ shifting to the right. The last $g-k-1$ rows (of blocks) have the identity matrix shifting to the right. Our formula for $M^{g}$ follows easily.

Since the spectral radius of a PF matrix is majorized by its greatest column sum (see [Ga]), we conclude that $\lambda^{g} \leq 11$, where $\lambda$ is the dilatation of $\phi$, and we have the estimate $\log \lambda \leq \log 11 / g$ provided $g \geq 4$. One checks directly 
that this bound also holds in the cases $g=2$ and $g=3$, and we conclude

Theorem. We have the estimates

$$
\frac{\log 11}{g} \geq l_{g}^{0} \geq \frac{\log 2}{12 g-12} .
$$

Remark. [Ba] can produce analogous examples of pA maps on $F_{g}^{0}$ and establish the upper bound $\log 6 / g \geq l_{g}^{0}$.

To close, we remark that it is likely to be a subtle problem to exhibit a pA map of $F_{g}^{s}$ realizing the infimal dilatation $l_{g}^{s}$.

\section{ACKNOWLEDGMENT}

Let me thank Athanase Papadopoulos for helpful discussions, Bill Thurston for a tea-time chat on this material, Max Bauer for corrections to an earlier version of this manuscript, and Bill Abikoff for a critical reading. It is a pleasure to acknowledge the kind support of Harvard University and Institut MittagLeffler during the Fall of 1989.

\section{REFERENCES}

[Ab] W. Abikoff, The real-analytic theory of Teichmüller space, Lecture Notes in Math., vol. 820, Springer-Verlag, 1980.

[AY] P. Arnoux and J. Yoccoz, Construction de diffeomorphisme pseudo-Anosov, C. R. Acad. Sci. Paris 292 (1981), 75-78.

[Ba] M. Bauer, Examples of pseudo-Anosov homeomorphisms, Thesis, Univ. of Southern California, 1989.

[FLP] A. Fathi, F. Laudenbach, , V. Poenaru et al., Travaux de Thurston sur les surfaces, Astérisque 66-67 (1979).

[Ga] F. Gantmacher, Theory of matrices, vol. 2, Chelsea, 1960.

[Pa] A. Papadopoulos, Reseaux Ferroviares, diffeomorphismes pseudo-Anosov et Automorphismes symplectiques de l'homologie, Publ. Math. d'Orsay 83-103 (1983).

[P1] R. C. Penner, A construction of pseudo-Anosov homeomorphisms, Trans. Amer. Math. Soc. 310 (1988), 179-197.

[PH] R. C. Penner with J. L. Harer, Combinatorics of train tracks, Ann. Math. Stud., Princeton Univ. Press, Princeton, NJ (to appear).

[PP] A. Papadopoulos and R. C. Penner, A characterization of pseudo-Anosov foliations, Pacific J. Math. 130 (1987), 359-377.

[Th] W. Thurston, The geometry and topology of three-manifolds, Ann. Math. Stud., Princeton Univ. Press, Princeton, NJ (to appear).

Department of Mathematics, University of Southern California, Los Angeles, CaliFORNIA 90089 